\title{
Extracellular matrix secreted by senescent fibroblasts induced by UVB promotes cell proliferation in HaCaT cells through PI3K/AKT and ERK signaling pathways
}

\author{
JIAN KANG $^{1 *}$, WENQI CHEN ${ }^{1 *}$, JIPING XIA $^{1}$, YANHUA LI $^{1}$, BO YANG ${ }^{1}$, BIN CHEN $^{1}$, WEILING SUN ${ }^{1}$, \\ XIUZU SONG ${ }^{2}$, WENZHONG XIANG ${ }^{2}$, XIAOYONG WANG $^{3}$, FEI WANG ${ }^{4}$, ZHIGANG BI $^{1}$ and YINSHENG WAN ${ }^{5}$ \\ ${ }^{1}$ Department of Dermatology, the First Affiliated Hospital of Nanjing Medical University, Nanjing 210029, Jiangsu Province; \\ ${ }^{2}$ Department of Dermatology, the Third Hospital of Hangzhou, Hangzhou 310009, Zhejiang Province; ${ }^{3}$ Department of \\ Dermatology, the First Affiliated Hospital, College of Medicine, Zhejiang University, Hangzhou 310003, Zhejiang \\ Province; ${ }^{4}$ Department of Dermatology, Zhongda Hospital of Southeast University, Nanjing 210009, Jiangsu \\ Province, P.R. China; ${ }^{5}$ Department of Biology, Providence College, Providence, RI 02918, USA
}

Received December 18, 2007; Accepted February 22, 2008

\begin{abstract}
Chronic exposure to solar ultraviolet radiation (UV) induces photoaging, and ultimately photocarcinogenesis. Senescent human skin fibroblasts (HSFs) in UVB stressinduced premature senescence (UVB-SIPS) share a similar extracellular matrix (ECM) phenotype with other types of senescent fibroblast. ECM from senescent fibroblasts induced by a variety of stresses has been shown to promote preneoplastic and neoplastic epithelial cell growth, a potential mechanism in carcinogenesis. We undertook this study to explore whether the extracellular matrices from UVB-induced senescent fibroblasts have any effect on the proliferation of HaCaT cells. The results showed that ECM secreted from HSFs in UVB-SIPS has 13.15 and $29.27 \%$ more stimulatory effect on proliferation than ECM secreted from presenescent HSFs and non-ECM, respectively. ECM from fibroblasts in UVB-SIPS activates FAK, ERK, and AKT in HaCaT cells. ERK and PI3K/AKT inhibitors inhibit ECM-induced ERK, AKT activation and cell proliferation. Cytochalasin D, a destructive agent of the cytoskeleton, inhibits ECM-induced FAK activation and cell proliferation
\end{abstract}

Correspondence to: Dr Zhigang Bi, Department of Dermatology, the First Affiliated Hospital of Nanjing Medical University, Nanjing 210029, Jiangsu Province, P.R. China

E-mail: eltonbi@21cn.com

Dr Yinsheng Wan, Department of Biology, Providence College, Providence, RI 02918, USA

E-mail: yswan@providence.edu

${ }^{*}$ Contributed equally

Key words: ultraviolet, extracellular matrix, senescence, extracellular signal-regulated kinase, AKT, tumorigenesis in $\mathrm{HaCaT}$ cells. Collectively, we conclude that ECM secreted from HSFs in UVB-SIPS promotes cell proliferation via ERK and PI3K/AKT pathways and modulation of FAK and cytoskeletal proteins in $\mathrm{HaCaT}$ cells. Pharmacological manipulation of those signaling components may lead to the prevention and treatment of skin cancer induced by chronic solar exposure.

\section{Introduction}

Chronic exposure of solar ultraviolet radiation (UV) induces photoaging, and ultimately photocarcinogenesis. UVB has been implicated as the main cause for skin cancer $(1,2)$. Pathologically, photoaging is characterized by the abnormal structure of the dermal connective tissue and alteration of dermal extracellular matrix (ECM) resulting from chronic UV irradiation (2). UVB crosses the epidermis, reaching the upper dermis composed mainly of fibroblast and ECM. Higher levels of senescent cells are found in fibroblasts from skin biopsies from older donors compared with younger counterparts (3). Moreover, ex vivo experiments have shown that the repeated exposure of human skin fibroblasts to UVB at subcytotoxic level triggers UVB stress-induced premature senescence (UVB-SIPS) (4).

Fibroblasts are well known for their functions in maintaining the homeostasis of ECM in tissues, which can be disturbed by repeated UV exposure. Over the past few decades, studies have focused on the mechanism through which UVB-induced premature senescence fibroblasts influence dermal ECM. Little has been known about how the alteration of ECM in photoaging skin affects the cellular activities of epithelial cells, such as survival, proliferation, cell cycle progression and relevant signal transduction pathways, despite the fact that all these processes have been implicated as essential mechanisms in the development of skin cancer, involving the clonal expansion of initiated cells giving rise to pre-malignant and then to malignant lesions $(5,6)$. 
Several reports have cast light on the relationship of senescence and tumorigenesis, suggesting that senescent fibroblasts induced by a variety of stresses promote preneoplastic and neoplastic epithelial cell growth, but not normal epithelial cell growth, by the secretion of dissoluble factors, the deposition of ECM and direct cell-cell interaction. Extracellular matrix deposited by senescent fibroblasts accounts for at least $40 \%$ of growth stimulation (7). Senescent human skin fibroblasts (HSFs) induced by successive UVB exposure also share a similar phenotype with other types of senescent fibroblasts, in which the increase of certain extra-cellular matrix components, such as collagens, fibronectin and osteonectin, have been found $(4,8,9)$. However, whether ECM deposited by HSFs in UVB-SIPS exhibits a similar effect on growth stimulation or cell proliferation remains unknown.

HaCaT cells are human preneoplastic epidermal keratinocytes, having acquired only some mutations that predispose to malignancy. Thus, it is a commonly used cell model for the understanding of the cellular and molecular mechanisms of tumorigenesis $(7,10)$. Understanding the effects of ECM on HaCaT cell proliferation may offer a better understanding of cell progression. Thus far, two wellestablished models have been available to investigate how the ECM secreted by persenescent HSFs and HSFs in UVBSIPS influence the functions of $\mathrm{HaCaT}$ cells. One is the induction of premature senescence fibroblasts by repeated UVB exposure (11), and another is the preparation of the extracellular matrix of fibroblasts (7).

Previous observations have suggested that ECM secreted by senescent fibroblasts can preferentially promote $\mathrm{HaCaT}$ cell proliferation and progression (7). Although, the mechanisms responsible for these effects have yet to be understood, ERK1/2 (12) and PI3K/AKT (13) signaling pathways have been implicated in the regulation of survival and proliferation in response to cell-ECM interaction in many types of cells (14). Focal adhesion kinase (FAK), a 125-kD tyrosine kinase, appears to also play a central role in integrin-mediated signal transduction, which normally localizes with surface integrin receptors, and is activated by cell binding to ECM (15), and PI3K/AKT and RAS-RAF-ERK1/2 are well-known downstream effectors of FAK (16-18). Activation of these pathways has been found to regulate cell survival and growth $(18,19)$.

Given all aforementioned information, we hypothesized that ECM secreted by HSFs promotes cell proliferation via the activation of ERK and PI3K/AKT pathways in HaCaT cells. Our results provide evidence to support this hypothesis. The modulation of these signaling pathways may lead to better prevention or clinical management of UV-induced skin aging and skin cancer.

\section{Material and methods}

Cell culture. Human skin fibroblasts (HSFs) at early cumulative population doublings (CDPs 25) were used for this experiment. HSFs were routinely cultivated in $175-\mathrm{cm}^{2}$ culture flasks containing $35 \mathrm{ml}$ of DMEM/F12 (Invitrogen, USA) supplemented with $10 \%$ fetal calf serum (FCS) (Invitrogen, AU). HaCaT cells were cultured in serum-free KBM medium, as previously described $(7,20,21)$. Cultures were grown at $37^{\circ} \mathrm{C}$ in a humidified atmosphere containing $5 \% \mathrm{CO}_{2}$. When $80 \%$ confluent, the cells were subcultured.

UVB irradiation and identification of HSFs in UVB-SIPS. UVB irradiation of fibroblast cultures was accomplished using a Philips SS-04P/UVB source (wavelength 290-320 nm). The intensity of the radiation from the UVB source was measured prior to each experiment using UVR radiometer with a UVB sensor (Bioblock Scientific, Belgium). As described previously (11), presenescent HSFs $\left(5 \times 10^{6}\right.$ cells) were subcultured $10-\mathrm{cm}$ culture dishes at $50 \%$ confluence in $\mathrm{DMEM} / \mathrm{F} 12+1 \%(\mathrm{v} / \mathrm{v}) \mathrm{FCS}$, and $2 \mathrm{mM}$ L-glutamine and $100 \mu \mathrm{g} / \mathrm{ml}$ streptomycin. At $72 \mathrm{~h}$ after plating, they were washed once with phosphate-buffered saline (PBS) $\mathrm{pH}$ 7.4, and exposed to UVB radiation in a thin layer of PBS with a dose of $150 \mathrm{~J} / \mathrm{m}^{2}$. After irradiation, PBS was replaced by DMEM/F12 + 1\% FCS. The radiation stress was performed once a day for 5 days. At 3 days after the last radiation, a senescent phenotype of HSFs was observed under a microscope. Presenescent HSFs were kept in the same culture conditions without UVB exposure. HSFs in UVB-SIPS were identified at 3 days after the last UVB stress. The fibroblasts treated or untreated were transferred to $35-\mathrm{mm}$ culture dishes at a density of 700 cells $/ \mathrm{cm}^{2}$ in DMEM/F12 + 1\% FCS.

Senescence-associated $\beta$-galactosidase (SA $\beta$-gal) activity assay. Activity was determined $24 \mathrm{~h}$ later as described previously (3). The population of SA B-gal-positive cells was determined by counting 400 cells per dish. The proportions of cells positive for SA $\beta$-gal activity were given as a percentage of the total number of cells counted in each dish. The results are expressed as the mean of triplicates \pm SD. Presenescent and senescent HSFs in UVB-SIPS were identified as containing $<10 \%$ and $>60 \%$ senescenceassociated SA B-gal-positive, respectively (4).

The preparation of extracellular matrix (ECM) deposited by HSF $s$ and the treatment of HaCaT cells. After identification, presenescent $\left(4 \times 10^{4} / \mathrm{cm}^{2}\right)$ and senescent $\left(5 \times 10^{4} / \mathrm{cm}^{2}\right)$ fibroblasts were allowed to attach to $3.5-$ or $6-\mathrm{cm}$ culture dishes overnight and were incubated in serum-free medium for 3-4 days to generate similar cell numbers. Cells were treated with $2 \mathrm{mM}$ EDTA for $3 \mathrm{~h}$ at $37^{\circ} \mathrm{C}$, followed by three rapid washes with PBS to remove cells, leaving the ECNM behind. Then, microscopic examination was performed to ensure the complete removal of fibroblasts and the presence of intact ECM in the wells or dishes (10). Fifty percent confluent HaCaT cells were incubated in growth factor-free KGM (Clonetics Co., USA) medium for 2 days, and detached with $0.05 \%$ trypsin and $0.05 \%$ EDTA. Trypsin activity was neutralized by $0.5 \%$ soybean trypsin inhibitor. HaCaT cells then were re-suspended in growth factor-free KGM medium which contained $\mathrm{KGM}, 1.8 \mathrm{mM} \mathrm{CaCl}, 5 \mathrm{mg} / \mathrm{ml}$ insulin and $0.5 \mathrm{mg} / \mathrm{ml}$ hydrocortisone, incubated at $37^{\circ} \mathrm{C}$ for $60 \mathrm{~min}$ in a rotator to allow intracellular signaling to basal level, and then plated on non-ECM dishes or dishes prepared above. In some studies, pharmacological agents were added to the HaCaT cells $1 \mathrm{~h}$ before seeding (22), while the cells were in suspension. The dose $(1 \mu \mathrm{M}$ of U0126, $400 \mathrm{nM}$ of wortmannin and $2 \mu \mathrm{M}$ of cytochalasin D, respectively) used in this experiment did 
not appear to have a conspicuous cytotoxic effect on HaCaT cultured routinely (MTT assay, data not shown). At the directed times, HaCaT cells were harvested according to the needs of the study.

Pharmacological agents. ERK1/2 inhibitor, U0126, PI3K inhibitor, wortmannin and cytochalasin D used in this study were obtained from Sigma (St. Louis, MO, USA).

Western blot analysis. To determine the time course of the tyrosine phosphorylation of proteins, $\mathrm{HaCaT}$ cells were plated at a density of 80,000 cells $/ \mathrm{cm}^{2}$ onto the ECM as prepared above or non-ECM as a control. At $0.5,1,2$ and $4 \mathrm{~h}$, the medium was gently aspirated, and the cells were scraped into cell lysis buffer [20 mM Tris ( $\mathrm{pH} \mathrm{8.0),} 150 \mathrm{mM} \mathrm{NaCl}$, $1 \%$ NP-40, $1 \%$ deoxycholate, $1 \mathrm{mM}$ EDTA, $1 \mathrm{mM}$ phenylmethylsulfonyl fluoride, $40 \mathrm{mM}$ glycerophosphate, $125 \mu \mathrm{M}$ $\mathrm{Na}_{3} \mathrm{VO}_{4}, 50 \mathrm{mM} \mathrm{NaF}, 2 \mu \mathrm{g}$ of leupeptin/ml, $2 \mu \mathrm{g}$ of aprotinin/ml, $2 \mu \mathrm{g}$ of pepstatin/ml, and $1 \mathrm{mM}$ dithiothreitol). One hundred microliters per 6 - $\mathrm{cm}$ dish of cell lysates were incubated in a cold room $\left(4^{\circ} \mathrm{C}\right)$ for $30 \mathrm{~min}$. After centrifugation at $14,000 \mathrm{x} \mathrm{g}$ for $10 \mathrm{~min}$ at $4^{\circ} \mathrm{C}, \mathrm{BCA}$ (Pierce, USA) assays were performed to determine total protein concentrations, which were normalized to $2 \mu \mathrm{g} / \mu 1$ for all samples. The samples $(30 \mu \mathrm{g})$ were then prepared in $6 \mathrm{X}$ SDS-PAGE sample loading buffer and denatured at $95^{\circ} \mathrm{C}$ for $5 \mathrm{~min}$, the protein was separated by $10 \%$ SDS-PAGE and electrotransferred to Immobilon-P membranes (Millipore, Bedford, MA, USA). After blocking with $3 \%$ bovine serum albumin (BSA) in Tris-buffered saline containing $0.1 \%$ Tween-20 (TBST), the membranes were incubated with primary antibody directed against total FAK, phospho-FAK (Tyr 397) (sc-11765-R, Santa Cruz Biotechnologies, USA), phosphoERK1/2 (E-4, Santa Cruz), phospho-Akt (Ser473) (Cell Signaling Technology, USA) and anti-GAPDH (Santa Cruz), in $2 \% \mathrm{BSA}$ in TBST overnight at $4^{\circ} \mathrm{C}$ or $1 \mathrm{~h}$ at $37^{\circ} \mathrm{C}$. After being washed three times with TBST, the membranes were then incubated with HRP-anti-mouse or rabbit (Santa Cruz) secondary antibody for $45 \mathrm{~min}$ at room temperature and subsequently washed. A chemiluminescent detection kit (Pierce) was then used to detect expression of these proteins and phospho-proteins, and the density of the bands was quantified using Quality One software (Bio-Rad, USA), as previously reported (23).

Cell proliferation assay. Cell proliferation was assessed by manual cell counting or MTT assay as previously reported $(20,21)$. Briefly, HaCaT cells $\left(1 \times 10^{5}\right.$ cells/well $)$ were seeded to $3.5-\mathrm{cm}$ dishes pre-coated with ECM prepared earlier or not, and treated as a control in EGF-free KBM medium. The medium was changed once every day. At 24, 48 and $96 \mathrm{~h}$ following seeding, attached $\mathrm{HaCaT}$ cells were harvested with trypsin and counted using a cell counter (Coulter, USA). For MTT assay $(20,21,24,25)$ at $96 \mathrm{~h}$ following seeding, the medium was changed to the same medium supplied with $0.8 \mathrm{mg} / \mathrm{ml}$ of MTT, and the HaCaT cells then were incubated for $4 \mathrm{~h}$. Washing with PBS was followed by the addition of $1 \mathrm{ml}$ of dimethylsulfoxide (DMSO, Sigma), shaking for $10 \mathrm{~min}$ so that complete dissolution was achieved. Aliquots $(150 \mu \mathrm{l})$ of the resulting solutions were transferred to 96 -well plates and absorbance was recorded at $560 \mathrm{~nm}$ using the Microplate Spectrophotometer System (Labsystems, Helsinki, Finland). In some experiments, pharmacological agents U0126, wortmannin and cytochalasin D were used as described above. Each experiment was repeated three times. No effects of either non-ECM or ECM deposited by both types of fibroblasts on stimulated apoptosis and proliferation was observed compared to DMSO as control.

Apoptosis assay. Apoptosis and cell cycle progression was evaluated $24 \mathrm{~h}$ after $\mathrm{HaCaT}$ cells being seeded with or without administration of the agents and determined with flow cytometry as described (26). Briefly, floating and adherent cells that were trypsinized were pooled and subsequently fixed in $80 \%$ ethanol $\left(-20^{\circ} \mathrm{C}\right)$ overnight. After being washed twice with PBS, cells were stained in PBS/ EDTA containing $7.5 \mathrm{mM}$ propidium iodide and $10 \mathrm{mg} / \mathrm{ml}$ RNase A. After 30 min incubation at room temperature, the cell cycle was analyzed by flow cytometry (FACScalibur, Becton Dickinson), and the percentages of cells in apoptosis were determined using the ModFit LT 3.0 program. Apoptosis assays were performed three times and recorded photographically every time.

Statistical analysis. All experiments were performed at least three times in triplicate and data was statistically analysed using SPSS 11.0 Software. The values in the figures are expressed as mean $\pm \mathrm{SD}$. Student's t-test was used to determine if there was a significant difference between two means $(\mathrm{p}<0.05)$, and statistical differences are indicated. When multiple means were compared, significance was determined by a one-way analysis of variance $(p<0.05)$ in conjunction with a Newman-Keuls post hoc test. For analysis of variance, letter designations are used to indicate significant differences. Means with a common letter designation are not different; those with a different letter designation are significantly different from all other means with different letter designations.

\section{Results}

Effect of different ECMs on the proliferation of HaCaT cells. To determine whether changes in ECM in response to repeated UVB exposure promote $\mathrm{HaCaT}$ cell proliferation, we treated HaCaT cells with ECM deposited by fibroblasts from different treatment. We preincubated HaCaT cells in growth factor-deficient KGM medium before seeding cells onto dishes coated by ECM secreted from HSFs in UVBSIPS and presenescent HSFs or non-treated as a control. Proliferation experiments were performed as described in Materials and methods. Our results showed that ECM secreted from HSFs in UVB-SIPS had 13.15 and $29.27 \%$ $(p<0.05)$ more stimulatory effect than ECM secreted from presenescent HSFs and non-ECM, respectively (Fig. 1). Interestingly, ERK inhibitor U0126, PI3K inhibitor wortmannin, and FAK inhibitor cytochalasin D, inhibited ECM-induced $\mathrm{HaCaT}$ cell proliferation.

Effects of different ECMs on cell-cycle progression and apoptosis of HaCaT cells. To further study whether the 
proliferation effect is due to lower apoptosis or higher propagation, flow cytometry analysis was performed to evaluate the effects of different ECM on cell-cycle progression and apoptosis. The results showed that compared with non-ECM groups, the groups cultured in ECM secreted from HSFs in UVB-SIPS and presenescent HSFs had a 23.68 and $8.55 \%$ increase of cell proportion in $\mathrm{S}$ phase $(\mathrm{p}<0.05)$ and a 6.23 and $6.20 \%$ decrease of cells in $\mathrm{G} 0 / \mathrm{G} 1$ phase, respectively, indicating that both ECM from HSFs in UVBSIPS and presenescent HSFs accelerated cell-cycle progression by promoting the G1-S phase transition, but, there was a greater effect of ECM from HSFs in UVB-SIPS (Fig. 2C). Unexpectedly, apoptosis analysis showed an opposite result: that $\mathrm{HaCaT}$ cultured in non-ECM, ECM from presenescent HSFs and HSFs in UVB-SIPS had averages of 3.88, 5.53 and $7.04 \%$ apoptosis, respectively (Fig. 2A and B). As expected, ERK inhibitor U0126, PI3K inhibitor wortmannin, and FAK inhibitor cytochalasin D exacerbated ECM-induced apoptosis (Fig. 2A and B).

Effect of different ECMs on the level of FAK, ERK1/2, and AKT phosphorylation. To determine the activation of FAK, ERK1/2, and AKT in HaCaT cells seeded on ECM deposited by fibroblasts from different treatments, we measured the phosphorylation of FAK, ERK, and AKT in time course experiments using phospho-specific antibodies. The results showed that attachment to prepared ECM (non-ECM controls, ECM deposited by HSFs in UVB-SIPS and ECM by presenescent HSFs) induced both FAK and ERK1/2 phosphorylation in a time-dependent manner, both FAK and ERK1/2 phosphorylation began at $30 \mathrm{~min}$, reached a peak at $120 \mathrm{~min}$, and remained elevated for $4 \mathrm{~h}$ (Fig. 3). Densitometric analysis data showed that peak phosphorylation of ERK1/2 in UVB-SIPS groups and presenescent groups was 177 and $131 \%$ of non-ECM controls $(\mathrm{p}<0.05)$, whereas FAK activity was 154 and $118 \%$, respectively. The most significant difference of FAK phosphorylation occurred at $240 \mathrm{~min}$; UVB-SIPS groups and presenescent groups exhibited 203\% (Fig. 3F) and 145\% of ERK phosphorylation $(\mathrm{p}<0.01)$ (Fig. 3D) compared to controls, respectively. Collectively, in three groups, ECM deposited by HSFs in UVB-SIPS induced the most rapid and intense phosphorylation of ERK1/2 and FAK. For AKT, attachment to nonECM and ECM deposited by HSFs in UVB-SIPS or by presenescent HSFs induced a slight but not significant phosphorylation of AKT (Fig. 3E).

The effects of ERK1/2 inhibitor U0126 and PI3K inhibitor wortmannin. The above data suggest that PI3K/AKT and ERK may be involved in ECM-mediated cell proliferation. $\mathrm{PI} 3 \mathrm{~K} / \mathrm{AKT}$ functions as a protective signaling against apoptosis, including anoikis, in different cell types, while, it remains controversial whether ERK functions as an antiapoptotic or a pro-apoptotic molecule (27). To further determine whether ERK1/2, and PI3K/AKT signaling pathway mediates this ECM-induced proliferation of $\mathrm{HaCaT}$ cells, we used selective ERK 1/2 inhibitor U0126 and PI3K inhibitor wortmannin. The results showed that U0126 and wortmannin completely blocked phosphorylation of ERK1/2 and $\mathrm{AKT}$ at a concentration of $1 \mu \mathrm{M}$ and $400 \mathrm{nM}$

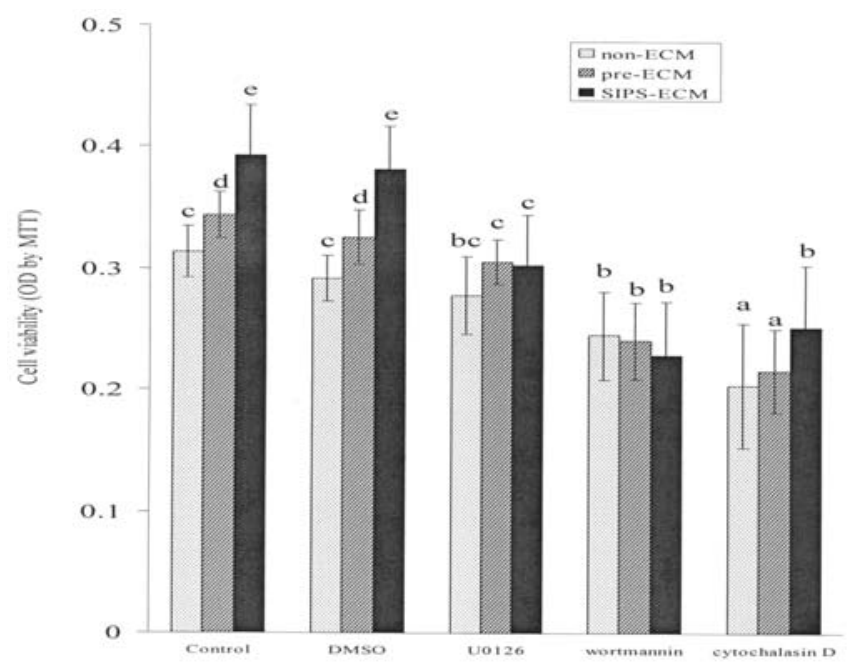

Figure 1. Effects of ECMs from different fibroblasts on the proliferation of $\mathrm{HaCaT}$ cells. Fifty percent confluent $\mathrm{HaCaT}$ cells were incubated in growth factor-free KBG medium for 2 days before suspension and being seeded onto the non-modified dishes and the dishes coated by ECM from HDFs in UVB-SIPS or presenescent HDFs, as described in Materials and methods. This was performed in control EGF-free KBM medium (blank), medium supplemented with a DMSO vehicle control, or the medium supplemented with ERK inhibitor U0126, PI3K inhibitor wortmannin or cytochalasin D, respectively. After 4 days incubation, MTT assay was performed to evaluate the effect of proliferation. The data are means \pm SD from one experiment representative of three. Letter designations above the bar denote statistical significance $(\mathrm{p}<0.05)$ when the bars are labeled with different letters. Where labeled with two or more letters, the data are not significantly different from other data labeled with any of the same letters.

respectively (Fig. 4). The treatment at such concentration did not induce vacuolization or otherwise affect the cells morphologically, at least at the light microscopic level (data not shown).

The effects of cytochalasin D. Previous studies have demonstrated that tyrosine phosphorylation of FAK in response to multiple stimuli requires an intact actin cytoskeleton. Specifically, treatment of the cells with cytochalasin D, which disrupts filamentous actin and promotes their depolymerization, completely inhibits FAK tyrosine phosphorylation (28). While the treatment with cytochalasin $\mathrm{D}(2 \mu \mathrm{M})$ did not induce cell detachment and slightly affected $\mathrm{HaCaT}$ cell morphology, addition of cytochalasin $\mathrm{D}$ to the medium for 4 days completely inhibited the effect of ECM-induced proliferation as shown in Fig. 1. To further study the effect of cytochalasin D, we used Western blot analysis. Treatment with $2 \mu \mathrm{M}$ cytochalasin D inhibited AKT phosphorylation and dramatically decreased FAK phosphorylation induced by cell-ECM attachment, but unexpectedly activated ERK1/2 (Fig. 5).

\section{Discussion}

Although UVB is thought to contribute to both skin aging and cancer, the interplay between photoaging and photocarcinogenesis has not been thoroughly studied. Repeated sublethal doses of UVB can induce premature senescence of 
A

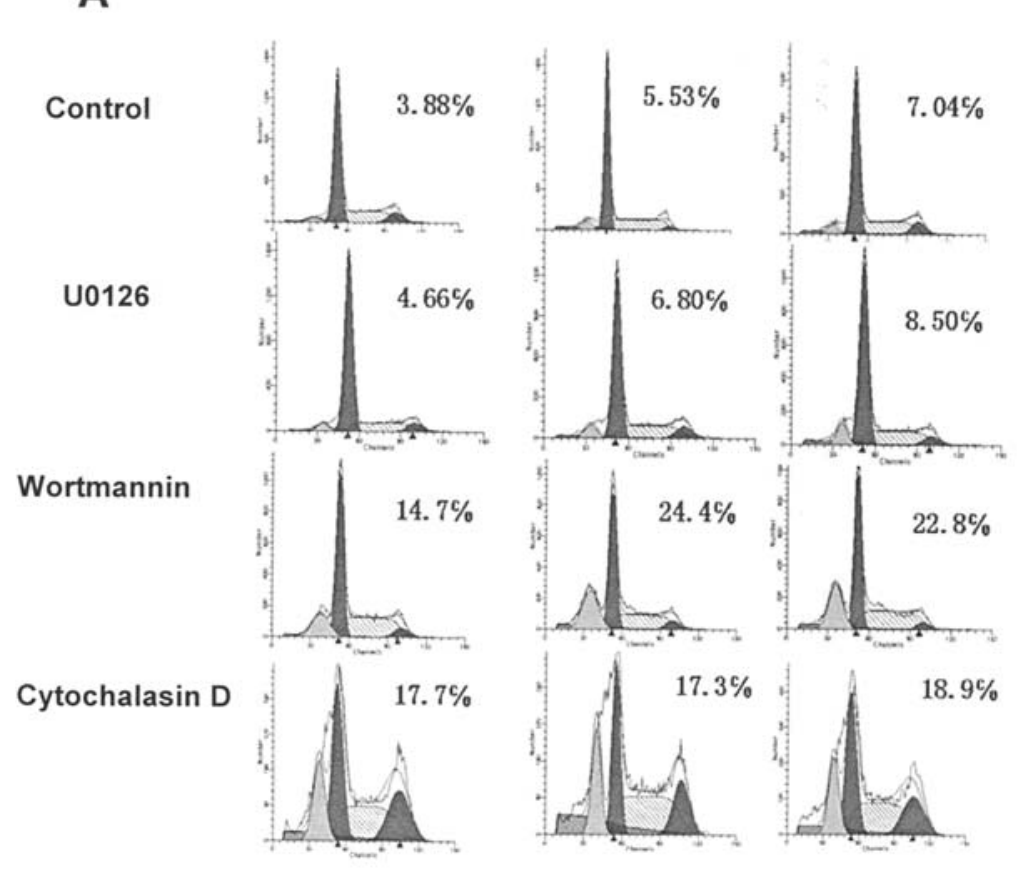

B
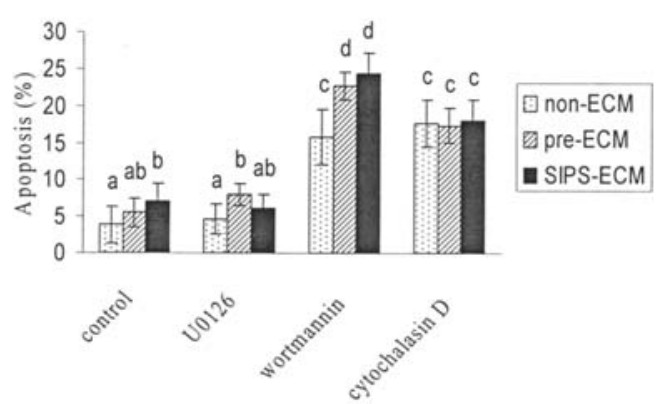

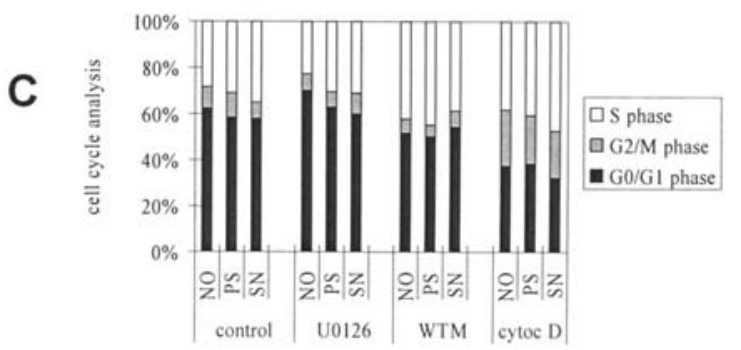

Figure 2. Effects of different ECMs on cell-cycle progression and apoptosis of HaCaT cells. (A) FACS analysis. HaCaT cells were prepared as above, with the presence of ERK inhibitor U0126, PI3K inhibitor wortmannin and cytochalasin D or DMSO control, were plated onto non-ECM dishes and the dishes were coated by ECM from HSFs in UVB-SIPS (SIPS-ECM) or presenescent HSFs (pre-ECM) in EGF-free KGM, respectively. At $24 \mathrm{~h}$ after being seeded, the floating and adherent cells were collected, fixed and PI stained, and FACS analysis was performed as described in Materials and methods. The rate of apoptosis was indicated as percentages of sub-G0/G1 phase cells. One representative experiment is illustrated. The numbers in the upper-right of each graph indicate the percentages of cells in apoptosis. (B) The average percentages of apoptosis. (C) Cell cycle analysis. The percentages of S-phase cells (white bars), G0/G1-phase cells (gray bars) and G2/M-phase cells (black bars) are shown. NO, non-ECM groups; PS, pre-ECM groups; SN, UVB-SIPS groups; WTM, wortmannin; cytoc D, cytochalasin D.

non-ECM

\section{A}

Oh $\quad 0.5 \mathrm{~h} \quad 1 \mathrm{~h} \quad 2 \mathrm{~h} \quad 4 \mathrm{~h}$

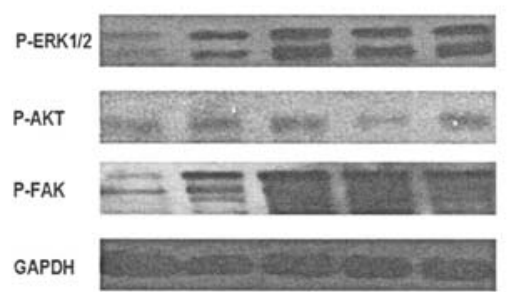

pre-ECM

B

oh $\quad 0.5 \mathrm{~h} \quad 1 \mathrm{~h} \quad 2 \mathrm{~h} \quad 4 \mathrm{~h}$

P-ERK1/2

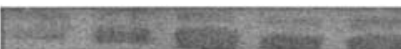

P-AKT

P-FAK

GAPDH
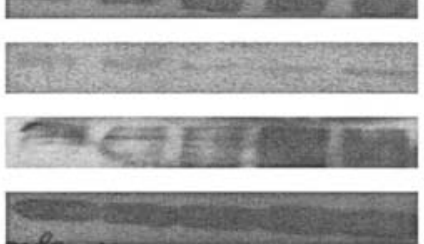

SIPS-ECM

\section{C}

Oh $\quad 0.5 \mathrm{~h} \quad 1 \mathrm{~h} \quad 2 \mathrm{~h} \quad 4 \mathrm{~h}$

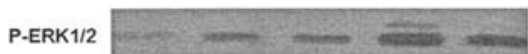

P-AKT

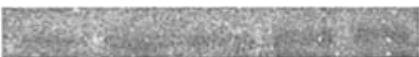

P-FAK

GAPDH
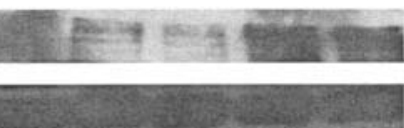

D

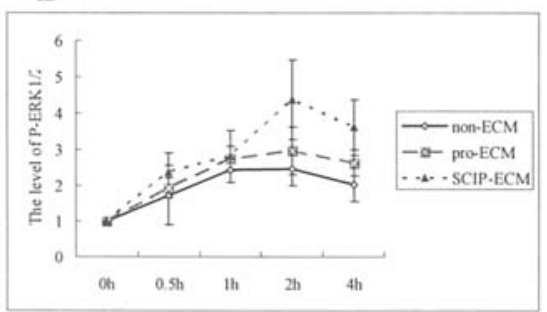

E

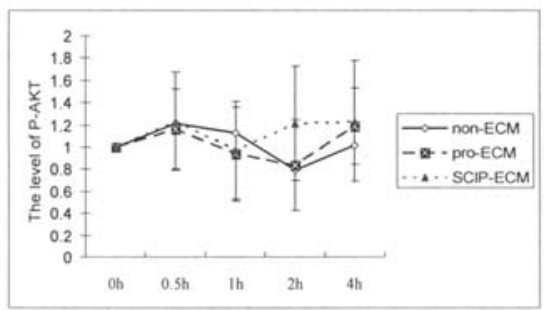

$\mathbf{F}$

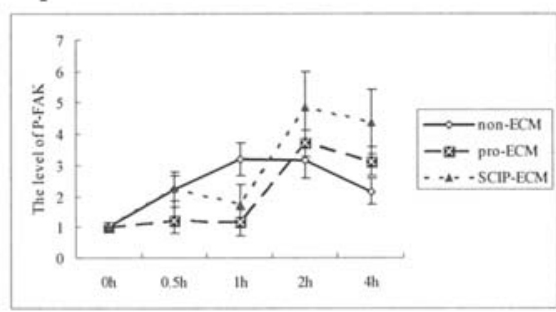

Figure 3. Effects of different ECMs on the levels of FAK, ERK1/2, and AKT phosphorylation. HaCaT cells were plated onto dishes coated by ECM from presenescent HSFs (pre-ECM) and from HSFs in SIPS-ECM (SIPS-ECM) prepared as described, or non-modified dishes (non-ECM). At of 0.5, 1, 2 and $4 \mathrm{~h}$, the cells were lysed, the proteins were separated by SDS-PAGE, probed with antibodies to phospho-ERK1/2, phospho-AKT, phospho-FAK and GAPDH and visualized using ECL [(A) non-ECM groups, (B) pre-ECM groups and (C) SIPS-ECM groups]. The densities of these bands were analyzed, and the values were quantified and normalized to the total amount of GAPDH. The time-course manner of ERK1/2, AKT and FAK phosphorylation was shown in D, E and $\mathrm{F}$, respectively. The data were from three independent experiments. 
A

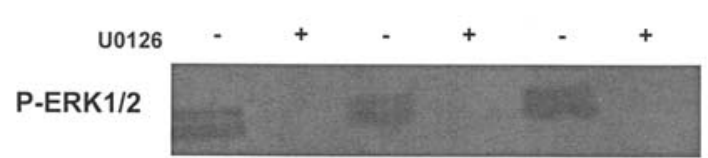

B

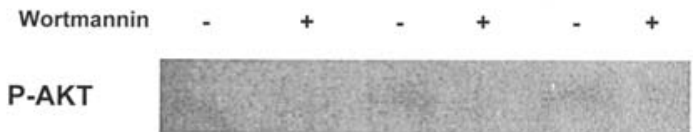

Figure 4. The effects of ERK1/2 inhibitor U0126 and PI3-K inhibitor wortmannin. HaCaT cells were kept in suspension and pretreated either in the absence or presence of ERK inhibitor U0126 or PI3K inhibitor wortmannin for $60 \mathrm{~min}$, then were seeded in the non-modified dishes (lanes 1 and 2), the dishes coated by ECM from presenescent HDFs (lanes 3 and 4) and the dishes coated by ECM from HDFs in UVB-SIPS (lane 5 and 6) in EGF-free KGM, respectively. At $2 \mathrm{~h}$ (for analysis of ERK) and $4 \mathrm{~h}$ (for AKT) after treatment, the cells were collected and lysed, and phosphorylation of ERK (A) and AKT (B) was analyzed by Western blotting with specific antibodies against phospho-ERK1/2, phospho-AKT.

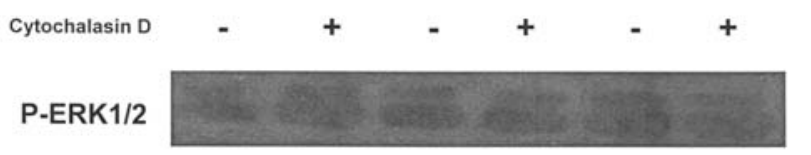

P-AKT

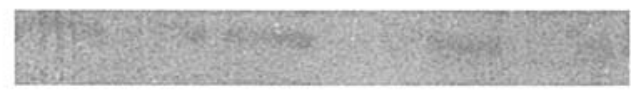

P-FAK

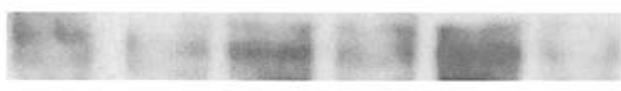

GAPDH

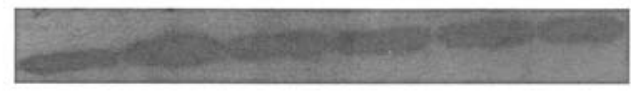

Figure 5. The effects of cytochalasin D. Pretreated HaCaT cells were plated onto dishes coated with prepared ECM or non-modified dishes either in the absence or presence of $2 \mathrm{M}$ cytochalasin $\mathrm{D}$, as described in Materials and methods. At $2 \mathrm{~h}$ after treatment, $\mathrm{HaCaT}$ cells were harvested and equal amounts of proteins were immunoblotted with antibodies to phosphoERK1/2, phospho-AKT, phospho-FAK and GAPDH.

HSFs, which undergo a change of ECM-secreted phenotype similar to that of other types of senescent fibroblasts $(4,8,9)$. ECM secreted by senescent human fibroblasts stimulated hyperproliferation and progression of preneoplastic epithelial cells, a potential mechanism for tumorigenesis (7). Whether a similar scenario occurs with ECM from HSFs in UVB stressinduced premature senescence (UVB-SIPS) and what the possible intracellular mechanisms involved are largely unknown. Our data demonstrate that ECM from HSFs in UVB-SIPS stimulates the proliferation of HaCaT cells compared to ECM from presenescent HSFs and controls, which is partially mediated by FAK and ERK, and in a PI3K/AKT-dependent manner.

The proliferation of $\mathrm{HaCaT}$ cells relies on cell survival and multiplication. Two mechanisms may be involved in the proliferation: the mechanism that rescues cells from apoptosis, and the mechanism that facilitates the growth of cells. Fibroblasts are known to produce collagens, fibronectins, and laminins which have been proved to be essential for cell survival and proliferation in various cell types including keratinocytes. HSFs in UVB-SIPS increase the expression of fibronectin and certain forms of collagens. Among other ECM components are fibronectin and collagen $(29,30)$ which function as rescuers from apoptosis and growth promoter. Although the hypothesis that ECM deposited by HSFs in UVB-SIPS can decrease apoptosis can be easily established, the result of the apoptosis assay described above showing that ECM from presenescent HSFs and HSFs in UVB-SIPS did not support more survival than non-ECM, seemingly does not support this. Indeed, one caveat is that ECM prepared as in our system is not composed of one single component. Among the components, some may act as rescuers, and others as inducers of apoptosis in specific cell types or experimental models. The rate of apoptosis in the presence of ECM derived differently may be the result of the coaction of these ECM components. Regardless of paradoxical results, our study showed that ECM secreted from HSFs in UVB-SIPS had 13 and 29\% more proliferation effects than ECM secreted from presenescent HSFs and nonECM, respectively (Fig. 1). Such proliferation effects were further supported by the results of the cell-cycle analysis, which indicated that ECM from HSFs in UVB-SIPS could more intensely promote the G1- to S-phase transition (Fig. 2).

Our results are in agreement with the findings that ECM produced by senescent fibroblasts was more stimulatory than matrix produced by presenescent fibroblasts in $\mathrm{HaCaT}$ cells, and that this effect of growth stimulation is independent of the senescence inducer (7). It is worth noting, however, that such a proliferation effect could be underestimated, both because the methods employed for preparing ECM may decrease and inactivate the native matrix components, and because the relative amount of initial cells decreases due to higher apoptosis in UVB-SIPS groups. Overall, all those data results suggest that ECM from HSFs in UVB-SIPS has more stimulatory effects on the proliferation of HaCaT cells, depending much more on higher multiplication than higher survival. These findings also support the notion that cellular senescence is an example of evolutionary antagonistic pleiotropy, protecting complicated organisms from cancer early in life, but promoting cancer later in life (31).

In this study, we also measured ERK1/2, AKT and FAK phosphorylation in response to different ECMs in HaCaT cells. The focus on ERK1/2, AKT and FAK is based not only on their significance in regulating multiple cell functions such as apoptosis and proliferation, but also on their unresolved roles in cell-ECM interaction in previous studies (32). Among MAP kinase family members, ERK is most commonly linked to the regulation of cell proliferation, while JNK and p38 are more strongly tied to stresses (33). The ERK pathway lies at the heart of many signal transduction processes and constitutes a major pathway through which many cell surface receptors including growth factor receptors and integrin receptors transduce their signals into the nucleus. Our study showed that blockade of ERK1/2 fully inhibits the effect of proliferation induced by ECM, which is consistent with many previous observations that ECM-cell attachment leads to substantial activation of ERK and then 
mediates proliferation effect. However, both positive and negative roles of ERK in cell survival regulation have been observed in several previous studies. In our model, the activation of ERK induced by cell-ECM interaction seemingly exhibited no effect on the apoptosis of HaCaT cells. Collectively, our results suggest that, activated ERK acts neither as an executioner of anoikis, nor as a survival factor, but likely acts as a promoter for the multiplication of $\mathrm{HaCaT}$. Thus, ERK may play different roles in apoptosis in different cell types and experiment models. What determines whether ERK acts in a pro-apoptotic or anti-apoptotic fashion remains an important but unanswered question.

The PI3K/AKT pathway is believed to transduce cell survival signals through the phosphorylation-dependent suppression of intracellular apoptotic factors such as BAD, GSK3 and IKK $\alpha$. Several studies have reported that environmental stresses capable of inducing apoptosis, such as UVC, ionizing radiation, and hyperosmotic stress lead to the downregulation of the PI3K/AKT pathway, and such downregulation is important in the apoptotic process. Treatment of cells with wortmannin blocks activation of AKT and increases cell death induced by various stresses (34). Our studies also showed that slight but detectable phosphorylated $\mathrm{AKT}$ was found in $\mathrm{HaCaT}$ cells under a condition of either attachment or suspension, and presented slight difference in the phosphorylated level among non-ECM groups, presenescent ECM groups and UVB-SIPS ECM groups (Fig. 3). Even though there were slight differences among the three groups, the phosphorylated form of AKT still worked as a critical protector of $\mathrm{HaCaT}$ cells from apoptosis in our system, the blockade of which could dramatically increase the apoptosis of cells and eventually inhibit the proliferation of HaCaT cells.

Our studies also showed that attachment to ECM rapidly and time-dependently stimulates FAK phosphorylation in HaCaT cells. FAK, a non-receptor PTK, has emerged as a critical link in integrin signal transduction as well as in the transduction of other signals, and has been implicated in the activation of AKT and ERK1/2 in many types of cells $(17,35)$. FAK may be involved in early signaling in response to cell-ECM interaction. To further explore the role of FAK activation in ERK and PI3K/AKT signaling in HaCaT cells subjected to attachment, we pretreated HaCAT cells with cytochalasin $\mathrm{D}$ which has been proved to induce filamentous actin depolymerization and completely inhibits FAK tyrosine phosphorylation in other types of cell.

Our results showed that $\mathrm{HaCaT}$ cells pretreated by $2 \mu \mathrm{M}$ cytochalasin D displayed a dramatic decrease of FAK and AKT activation in response to attachment and notably, an increase in ERK activation which was observed in previous reports (32). Parallel to the blockade of AKT activation, treatment with cytochalasin D dramatically decreased the rate of survival in the three groups, resembling the results of wortnaninn-treated groups. A proliferation assay indicated that treatment with cytochalasin D completely inhibited the stimulatory effect of ECM even though it appears to accelerate the cell-cycle progression (Figs. 1 and 2). It is obvious that the higher apoptosis induced by cytochalasin D overwhelmed such progression, further suggesting that AKT and ERK are both involved in the stimulatory effects of
ECM, the former acting as a rescuer, while the latter likely acting as a promoter of proliferation.

In conclusion, our data demonstrate that ECM from HSFs in UVB-SISP has more stimulatory effects on the proliferation of $\mathrm{HaCaT}$ cells, which appear to rely much more on higher multiplication than higher survival. In our system, low but detectable PI3K/AKT signaling can partially rescue $\mathrm{HaCaT}$ cells from apoptosis induced by EGFwithdrawing plus suspension, while attachment to ECM from HSFs of UVB-SIPS activates FAK and ERK1/2 signaling pathways which play an important role in promoting the multiplication of $\mathrm{HaCaT}$ cells, but not in enhancing cell survival. Our data provide insights into the understanding of the molecular mechanism involved in the stimulatory effects of UVB-SIPS ECM on HaCaT cell proliferation and further suggest that the modulation of AKT, ERK and FAK pathways could offer better clinical management or prevention of photocarcinogenesis.

\section{Acknowledgments}

This research was supported in part by grants from the National Natural Science Foundation of China (nos. 30271195 and 30671894), and by a grant from the NIH (P20 RR016457 from the INBRE Program of the National Center for Research Resources).

\section{References}

1. Scharffetter-Kochanek K, Wlaschek M, Brenneisen P, Schauen M, Blaudschun R and Wenk J: UV-induced reactive oxygen species in photocarcinogenesis and photoaging. Biol Chem 378: 12471257, 1997.

2. Fisher GJ, Kang S, Varani J, et al: Mechanisms of photoaging and chronological skin aging. Arch Dermatol 138: 1462-1470, 2002.

3. Dimri GP, Lee X, Basile G, et al: A biomarker that identifies senescent human cells in culture and in aging skin in vivo. Proc Natl Acad Sci USA 92: 9363-9367, 1995.

4. Debacq-Chainiaux F, Borlon C, Pascal T, et al: Repeated exposure of human skin fibroblasts to UVB at subcytotoxic level triggers premature senescence through the TGF-beta1 signaling pathway. J Cell Sci 118: 743-758, 2005.

5. Afaq F, Adhami VM and Mukhtar H: Photochemoprevention of ultraviolet B signaling and photocarcinogenesis. Mutat Res 571: 153-173, 2005.

6. Hussein MR: Ultraviolet radiation and skin cancer: molecular mechanisms. J Cutan Pathol 32: 191-205, 2005.

7. Krtolica A, Parrinello S, Lockett S, Desprez PY and Campisi J: Senescent fibroblasts promote epithelial cell growth and tumorigenesis: a link between cancer and aging. Proc Natl Acad Sci USA 98: 12072-12077, 2001

8. Dumont P, Burton M, Chen QM, et al: Induction of replicative senescence biomarkers by sublethal oxidative stresses in normal human fibroblast. Free Radic Biol Med 28: 361-373, 2000.

9. Gonos ES, Derventzi A, Kveiborg M, et al: Cloning and identification of genes that associate with mammalian replicative senescence. Exp Cell Res 240: 66-74, 1998.

10. Dilley TK, Bowden GT and Chen QM: Novel mechanisms of sublethal oxidant toxicity: induction of premature senescence in human fibroblasts confers tumor promoter activity. Exp Cell Res 290: 38-48, 2003.

11. Chainiaux F, Magalhaes JP, Eliaers F, Remacle J and Toussaint O: UVB-induced premature senescence of human diploid skin fibroblasts. Int J Biochem Cell Biol 34: 1331-1339, 2002.

12. Morino N, Mimura T, Hamasaki K, et al: Matrix/integrin interaction activates the mitogen-activated protein kinase, p44erk-1 and p42erk-2. J Biol Chem 270: 269-273, 1995.

13. Yamamoto D, Sonoda Y, Hasegawa M, Funakoshi-Tago M, Aizu-Yokota E and Kasahara T: FAK overexpression upregulates cyclin D3 and enhances cell proliferation via the PKC and PI3-kinase-Akt pathways. Cell Signal 15: 575-583, 2003. 
14. Clark EA and Brugge JS: Integrins and signal transduction pathways: the road taken. Science 268: 233-239, 1995.

15. Gabarra-Niecko V, Schaller MD and Dunty JM: FAK regulates biological processes important for the pathogenesis of cancer. Cancer Metastasis Rev 22: 359-374, 2003.

16. Asthagiri AR, Nelson CM, Horwitz AF and Lauffenburger DA: Quantitative relationship among integrin-ligand binding, adhesion, and signaling via focal adhesion kinase and extracellular signalregulated kinase 2. J Biol Chem 274: 27119-27127, 1999.

17. Sonoda Y, Watanabe S, Matsumoto Y, Aizu-Yokota E and Kasahara T: FAK is the upstream signal protein of the phosphatidylinositol 3-kinase-Akt survival pathway in hydrogen peroxide-induced apoptosis of a human glioblastoma cell line. J Biol Chem 274: 10566-10570, 1999.

18. Shen TL and Guan JL: Differential regulation of cell migration and cell cycle progression by FAK complexes with Src, PI3K, Grb7 and Grb2 in focal contacts. FEBS Lett 499: 176-181, 2001.

19. Schlaepfer DD and Hunter T: Signal transduction from the extracellular matrix - a role for the focal adhesion proteintyrosine kinase FAK. Cell Struct Funct 21: 445-450, 1996.

20. Cao C, Healey S, Amaral A, et al: ATP-sensitive potassium channel: a novel target for protection against UV-induced human skin cell damage. J Cell Physiol 212: 252-263, 2007.

21. Cao C, Sun Y, Healey S, et al: EGFR-mediated expression of aquaporin-3 is involved in human skin fibroblast migration. Biochem J 400: 225-234, 2006.

22. Chetoui N, Gendron S, Chamoux E and Aoudjit F: Collagen type I-mediated activation of ERK/MAP Kinase is dependent on Ras, Raf- 1 and protein phosphatase $2 \mathrm{~A}$ in Jurkat T cells. Mol Immunol 43: 1687-1693, 2006.

23. Li Y, Bi Z, Yan B and Wan Y: UVB radiation induces expression of HIF-1 $\alpha$ and VEGF through the EGFR/PI3K/DEC1 pathway. Int J Mol Med 18: 713-719, 2006.

24. Weichert H, Blechschmidt I, Schroder S and Ambrosius H: The MTT-assay as a rapid test for cell proliferation and cell killing: application to human peripheral blood lymphocytes (PBL). Allerg Immunol (Leipz) 37: 139-144, 1991.
25. Gurjala AN, Liu WR, Mogford JE, Procaccini PS and Mustoe TA Age-dependent response of primary human dermal fibroblasts to oxidative stress: cell survival, pro-survival kinases, and entrance into cellular senescence. Wound Repair Regen 13: 565-575, 2005.

26. Lacombe F and Belloc F: Flow cytometry study of cell cycle, apoptosis and drug resistance in acute leukemia. Hematol Cell Ther 38: 495-504, 1996.

27. Brognard J, Clark AS, Ni Y and Dennis PA: Akt/protein kinase $\mathrm{B}$ is constitutively active in non-small cell lung cancer cells and promotes cellular survival and resistance to chemotherapy and radiation. Cancer Res 61: 3986-3997, 2001.

28. Chen Q, Kinch MS, Lin TH, Burridge K and Juliano RL: Integrin-mediated cell adhesion activates mitogen-activated protein kinases. J Biol Chem 269: 26602-26605, 1994.

29. Gendron S, Couture J and Aoudjit F: Integrin alpha2beta1 inhibits Fas-mediated apoptosis in T lymphocytes by protein phosphatase 2A-dependent activation of the MAPK/ERK pathway. J Biol Chem 278: 48633-48643, 2003.

30. Maehata Y, Takamizawa S, Ozawa S, et al: Type III collagen is essential for growth acceleration of human osteoblastic cells by ascorbic acid 2-phosphate, a long-acting vitamin $\mathrm{C}$ derivative. Matrix Biol 26: 371-381, 2007.

31. Campisi J: Aging, tumor suppression and cancer: high wire-act! Mech Ageing Dev 126: 51-58, 2005.

32. Barberis L, Wary KK, Fiucci G, et al: Distinct roles of the adaptor protein $\mathrm{Shc}$ and focal adhesion kinase in integrin signaling to ERK. J Biol Chem 275: 36532-36540, 2000.

33. Kolch W: Meaningful relationships: the regulation of the Ras/Raf/MEK/ERK pathway by protein interactions. Biochem J 351: 289-305, 2000.

34. Meier R and Hemmings BA: Regulation of protein kinase B. J Recept Signal Transduct Res 19: 121-128, 1999.

35. Li W, Duzgun A, Sumpio BE and Basson MD: Integrin and FAK-mediated MAPK activation is required for cyclic strain mitogenic effects in Caco-2 cells. Am J Physiol Gastrointest Liver Physiol 280: G75-G87, 2001. 